
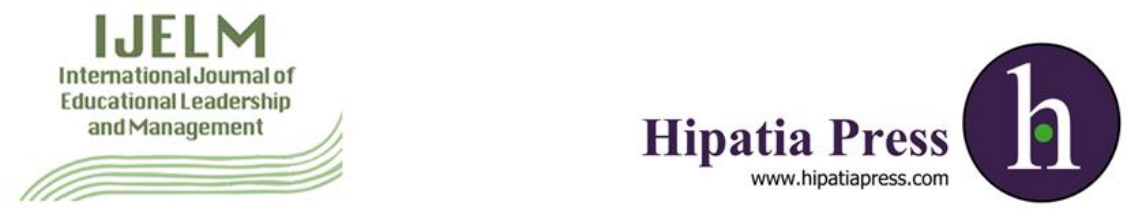

Instructions for authors, subscriptions and further details:

http://ijelm.hipatiapress.com

\title{
Prácticas de liderazgo intermedios en organizaciones escolares de Chile
}

Guillermo Andrés Rodríguez Molinaํ \& Joaquín Gairín Sallán²

1) Universidad de Concepción, Chile.

2) Universitat Autónoma de Barcelona, Spain.

Date of publication: January $16^{\text {th }}, 2020$

Edition period: July 2019 - January 2020

To cite this article: Rodríguez, G.A. \& Gairín, J. (2020). Prácticas de liderazgo intermedios en organizaciones escolares de Chile. International Journal of Educational Leadership and Management, 8(1), 88-111. doi: 10.17583/ijelm.2020.4044

To link this article: http://dx.doi.org/10.17583/ijelm.2020.4044

\section{PLEASE SCROLL DOWN FOR ARTICLE}

The terms and conditions of use are related to the Open Journal System and to Creative Commons Attribution License (CCAL). 


\section{Prácticas de liderazgo intermedios en organizaciones escolares de Chile}

Guillermo Andrés Rodríguez Molina

Universidad de Concepción

Chile
Joaquín Gairín Sallán

Universitat Autónoma de Barcelona

Spain

\section{Resumen}

Esta contribución tiene por objetivo describir los liderazgos intermedios de las organizaciones escolares, sus prácticas y como se asocian con las prácticas docentes. Principalmente, en el caso de Chile se diferencia el liderazgo escolar en uno administrativo (director de la organización) y otro pedagógico (líder intermedio "LI"). Se utilizó un método mixto de características exploratorias, lo que permitió realizar una descripción y relación entre las prácticas del liderazgo intermedio. Entre los resultados se encontró una alta tasa de prácticas pedagógicas que implementan los líderes intermedios por sobre los directores, y relaciones directas entre lo que hace el LI y los docentes de aula. Lo anterior, llevó a concluir que desarrollando planes pedagógicos con fuerte alcance en lo que realizan los docentes y estos líderes se alcanzarían resultados sobre la media en los procesos de evaluación docente y principalmente que los estudiantes logren aprendizajes con énfasis en el desarrollo de habilidades superiores del pensamiento.

Palabras claves: Lider intermedio, liderazgo escolar, practices pedagógicas, organización escolar. 


\section{Intermediate leadership practices in school organizations in Chile}

Guillermo Andrés Rodríguez Molina

Universidad de Concepción

Chile
Joaquín Gairín Sallán

Universitat Autónoma de Barcelona

Spain

\section{Abstract}

This contribution aims to describe the intermediate leadership of school organizations, their practices and how they are associated with teaching practices. Mainly, in the case of Chile, the school leadership is differentiated into an administrative one (director of the organization) and another pedagogical one (intermediate leader "LI"). A mixed method of exploratory characteristics was used, which allowed a description and relationship between intermediate leadership practices. Among the results was a high rate of pedagogical practices implemented by the intermediate leaders over the principals, and direct relationships between what the LI does and the classroom teachers. This led to the conclusion that developing pedagogical plans with a strong scope in what teachers and these leaders do would achieve results on the average in teacher evaluation processes and mainly that students achieve learning with an emphasis on the development of superior teaching skills.

Keywords: Intermediate leader, school leadership, pedagogical practices, school organization. 


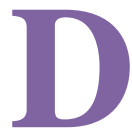

urante años se ha dicho que los buenos liderazgos escolares son claves para las organizaciones escolares, ya que pueden influir de manera nutritiva o tóxica en toda la institución por su fuerte permeabilización en todos los ámbitos de gestión escolar (Törnsén, 2014; Triviños, 2013; Bolívar, 2010).

En los primeros años de la década del 2000 la evidencia proveniente, principalmente, de USA y otros países de Europa (Weinstein y Hernández, 2015) mostraron que las prácticas de liderazgo directivo podían tener un impacto en los resultados de aprendizaje de los estudiantes. Concluyendo, que el liderazgo escolar sería el segundo factor intra escuela, después de los profesores, en impactar positiva o negativamente en los resultados de los estudiantes (Leithwood, Day, Sammons, Harris y Hopkins, 2006). Sin embargo, recientes estudios realizados por el Laboratorio Latinoamericano de Evaluación de la Calidad de la Educación (LLECE, 2016) señalan que no se muestra una asociación generalizada entre el liderazgo directivo con los resultados de aprendizaje de los estudiantes (Organización de las Naciones Unidas para la Educación, la Ciencia y la Cultura [UNESCO], 2016).

En este contexto, el liderazgo escolar desde hace una década está transitando hacia un cambio de paradigma necesario, el cual, deja el tradicional accountability originado en las escuelas efectivas con liderazgos en la gestoría de las organizaciones escolares o educational management (Bolívar, 2015; Hargreaves y Fullan, 2014; Leithwood y Levin, 2005) a comunidades de aprendizaje, donde los liderazgos de carácter pedagógico o educational leadership están enfocados en aspectos curriculares y pedagógicos, con énfasis en el desarrollo de las capacidades docentes (Rodríguez y Gairín, 2017; Hargreaves y Fullan, 2014; Bush, 2011; Gairín, 2003).

Así, autores como Male y Palaiologou (2012) distinguen entre "liderazgo centrado en el aprendizaje", más limitado a productos, y "liderazgo pedagógico", como un enfoque más holístico y comprensivo, centrado en sostener entornos efectivos de aprendizaje. Como bien lo definieron Murphy, Elliott, Goldring, y Porter (2007): 
"La piedra de toque de este tipo de liderazgo incluyen la capacidad de los líderes para (a) permanecer siempre centrado en lo que es clave: organización de la escuela, el aprendizaje, la enseñanza, el currículo y la evaluación; y (b) hacer que todas las otras dimensiones del centro escolar (por ejemplo, administración, organización, finanzas) están al servicio de una organización más robusta para mejorar el aprendizaje de los estudiantes" (p. 179).

Con esta distinción de los liderazgos, de manera transversal se evidencia la existencia de un equipo u otros liderazgos que comparten con el director las tareas o bien directamente asumiendo como principal responsable de las funciones técnico-pedagógicas, ya sea, bajo el cargo de subdirector o vicedirector en México, Ecuador, Argentina y República Dominicana; de coordinador o coordinador docente en el caso de Ceará/Brasil y República Dominicana; o de jefe de la unidad técnico pedagógica (UTP) en Chile (UNESCO, 2014), jefes de estudio en España o líderes intermedios en países del norte de Europa (Comisión Europea, 2013; Álvarez, 2003).

A nivel internacional, la discusión indica los desafíos de distribuir y planificar la sucesión del liderazgo, sosteniendo y proyectando los esfuerzos de mejora, dando cuenta tanto de la relevancia de la formación progresiva y certificación pre-servicio de líderes, como del reconocimiento de líderes intermedios y docentes lideres en la organización escolar (Galdames y Rodríguez, 2010). Ejemplo de ello, es Finlandia, que a partir de la reforma curricular entrada en vigencia en el año 2016, es por cuanto se hizo necesaria la separación de los liderazgos al interior de las instituciones escolares, uno con énfasis en la gestión pedagógica del currículum y otro en gestión administrativa del centro (Porkka, 2014). 


\section{Antecedentes conceptuales}

El liderazgo pedagógico en Chile de acuerdo a varios estudios sobre el desarrollo de competencias y prácticas directivas no está radicado en los directores y directoras de los centros (Rodríguez y Gairín, 2017; Bellei,Vanni y Valenzuela, 2015; OCDE, 2014; UNESCO, 2014; Rodríguez, 2011). Sino que, en la figura de un liderazgo intermedio dentro de la jerarquía institucional, el Jefe de la Unidad Técnica Pedagógica (en adelante JUTP). Este liderazgo, emerge en la década de los años 80, para fortalecer la función directiva y ampliarla para quienes participaban de ella, explicitando la importancia del liderazgo pedagógico, alojándolo en esta figura (Núñez, Weinstein y Muñoz, 2010).

¿Quiénes son los líderes intermedios y cuál es su rol dentro de las organizaciones escolares?

El concepto de liderazgo intermedio ha ido ganando creciente importancia dentro de la investigación asociada al liderazgo educativo. Múltiples estudios han mostrado el efecto beneficioso que producen los docentes que ejercen un liderazgo pedagógico entre sus pares, al influir en la mejora de las prácticas pedagógicas de los docentes, y colaborando activamente en la implementación de las reformas educativas en las escuelas (Supovitz, Sirinides y May, 2009; York-Barr y Duke, 2004).

No obstante lo anterior, una de la dificultades que ha debido enfrentar la investigación y el desarrollo de programas de formación en este ámbito, es justamente la ausencia de una definición clara y unívoca respecto a quienes son los líderes intermedios y cómo se puede comprender por tanto el concepto de liderazgo docente (York-Barr y Duke, 2004). En algunos casos, los docentes designados como líderes poseen roles formales de liderazgo, como por ejemplo, jefes de departamento, mentores o coordinadores académicos, mientras que en otros se entienden como docentes cuyo principal rol está asociado al ejercicio de la docencia en aula, pero que colaboran o han asumido de manera adicional responsabilidades asociadas al ejercicio directivo. 
Los liderazgos intermedios, en los cuales se posiciona el JUTP, tanto por su génesis como por requerimientos internos de la organización basan su trabajo en distintas dimensiones y tareas (MINEDUC, 2015; OCDE/CEPPE, 2013; Elmore, 2010; Pont et al., 2008), entre las cuales se pueden mencionar:

- La construcción de equipos de trabajo docente.

- La construcción de redes formales e informales para retroalimentar y apoyar la labor profesional.

- El asesoramiento o coaching para fomentar la mejora de la instrucción y el desarrollo profesional de sus pares.

- Colaborar en la generación de climas de confianza, participación y compromiso.

- Facilitar y otorgar entendimiento respecto a los cambios organizacionales y la implementación de acciones propias de las reformas educativas.

- Generar diálogos e interacciones centradas en las problemáticas propias de la enseñanza y el aprendizaje.

- Confrontar barreras y promover cambios culturales y estructurales que vayan en beneficio del quehacer docente y la mejora de la enseñanza.

- Colaborar y asesorar a los equipos directivos en la toma de decisiones.

Leithwood (2016), indica que los líderes medios presentan un rol estratégico para la mejora escolar. Esto, porque cuentan con un alto potencial para impactar en los centros escolares a través del liderazgo pedagógico y formativo (Wahlstrom y Seashore, 2012; Weller, 2001). Podemos señalar que las prácticas de estos líderes medios, tienen la potencialidad de contribuir al desarrollo y mejoramiento de prácticas de enseñanza de sus colegas, incidiendo más directamente en las prácticas de los docentes y por tanto en el aprendizaje de los alumnos (Grootenboer et. al, 2015; Bennett; 2009; Busher y Harris, 2001). 
Estos líderes pedagógicos medios, presentan una posición única para influir en la calidad de la enseñanza y el aprendizaje dentro de sus áreas temáticas. Como jefes de equipos tienen una poderosa influencia sobre las prácticas de aula, y son importantes agentes para el cambio y desarrollo dentro de sus áreas (Busher y Harris, 2001), Fitzgerald (2009) señala que estos liderazgos deben dedicarse tanto, al proceso de enseñanza y aprendizaje, como al trabajo de estar a cargo de un equipo de docentes.

Estos líderes pedagógicos pueden otorgar dirección estratégica y desarrollo de la asignatura, potenciar el proceso de enseñanza aprendizaje, liderar y gestionar al personal, y optimizar el empleo efectivo y eficiente de personal y recursos (Flückiger, Lovett, Dempster y Brown, 2015). Flückiger et al. (2015), señalan que estos liderazgos deben enfocarse en desarrollar personas y en ser líderes del aprendizaje para otros. En el mismo sentido, las relaciones de liderazgo escolar y profesores tendrían una influencia, examinando el potencial de colaboración activa en torno a asuntos de instrucción para el conferir la calidad de la enseñanza y el rendimiento de los estudiantes (Sans-Martín, Guàrdia, y Triadó-Ivern, 2016).

\section{Método}

\section{Diseño y preguntas de la investigación}

La investigación se abordó desde un enfoque cuantitativo, no experimental, descriptivo y exploratoria (Burch y Heinrich, 2015; Creswell, 2014; Hernández, Fernández, y Baptista, 2014). Los datos utilizados se obtuvieron de la muestra de la tesis doctoral, que el autor principal finalizó en junio de 2017, relacionada con la influencia de las prácticas de gestión curricular de los JUTP sobre las prácticas docentes. Puntualmente, los datos reportados en este artículo fueron recolectados mediante un cuestionario autoadministrado direccionado sólo a los JUTP, durante los meses de marzo a mayo de 2016 a 576 Jefes de Unidades Técnicas Pedagógicas de 
establecimientos municipales de las regiones Bío Bío y Araucanía-Chile. Lo cual equivale al $41.8 \%$ del total de JUTP de ambas regiones.

Las preguntas de investigación que orientaron el estudio son:

¿Qué prácticas implementan los JUTP cómo líderes intermedios?

¿Cuál es la frecuencia de implementación de las prácticas de gestión curricular?

¿Quién o quiénes son los responsables de implementación de las prácticas?

\section{Participantes, instrumento y procedimiento}

Para obtener la base de datos y de contacto de quienes fueron los sujetos que reportaron la información, se obtuvieron desde los directorios de los Departamentos de Administración de Educación Municipal de las 86 comunas y del Ministerio de Educación. Se llegó a obtener la población, dada por el número de establecimientos educacionales municipales, que fueron 1381 que equivalen al 26,1\% del total nacional. Específicamente, se enviaron 1376 (874 Bío Bío y 502 Araucanía) cuestionarios de los cuales se tuvo una tasa de respuesta del $41,8 \%$, quedando el $\mathrm{N}=576$.

El instrumento utilizado para recolectar la información correspondió a un cuestionario con preguntas dicotómicas, politómicas, de respuestas múltiples y abiertas. Estuvo constituido por 25 preguntas cerradas y 2 preguntas abiertas, distribuyéndose en 4 apartados: Información general, La unidad educativa, Las funciones de la Unidad Técnico Pedagógica, Evaluación del profesorado. Dicho instrumento se sometió a un proceso a través de la validez de contenido, donde se trató de determinar hasta dónde los ítems de un instrumento son representativos del dominio o universo de contenido de la propiedad que se desea medir (García y Cabero, 2011; Corral, 2009; Tejada y Giménez, 2006; Del Rincón et al., 1995), para ello los jueces expertos, en aspectos metodológicos como temáticos, que participaron de la validación fueron 10, todos con grado de doctor de distintas universidades de Chile y España. 


\section{Análisis de los datos}

Este proceso se realizó, en primer lugar, realizando una modelización de los datos para hacerlos compatible al programa informático SPSS v.22. Se determinaron 42 elementos para su análisis, obteniendo un Alfa de Cronbach de 0.802 como consistencia interna.

Luego, se procedió a obtener la frecuencia y descriptores para su análisis socio demográfico. A continuación, se aplicaron las pruebas estadísticas Chi-cuadrado para examinar la relación entre las variables analizadas, a través, de las tablas de contingencia, (del tipo categóricas o nominales) y por otra, la distribución que posee una variable, contrastando así los supuestos de asociación entre ellas. Para el caso de las variables ordinales, se utilizó la prueba Kruskal-Wallis, ya que lo aleatorio de la muestras (JUTP y docentes), al no requerir supuestos de normalidad permitieron contrastar las variables de acuerdo a su población. Finalmente, y siguiendo las características de la muestra, la cual no sigue ley normal, se aplicó a prueba de correlación de Spearman (Rho de Spearman), para establecer en cierta medida la influencia de las prácticas de gestión curricular sobre las prácticas pedagógicas docentes, entre otras.

\section{Limitaciones del estudio}

En el contexto del estudio se presentaron limitaciones en tres áreas: ampliar el espectro de discusión con otros investigadores del tema, la escasa información teórica y empírica con los sujetos de estudio, ya que la mayoría de la información existente tiene como foco los líderes de establecimientos educacionales y finalmente la combinación de diseños pudo tender a la perdida la objetividad por su contacto permanente con el objeto de estudio. 


\section{Resultados}

\section{Sobre las practicas de los líderes intermedios y su frecuencia}

De acuerdo, a la evidencia descriptiva se obtuvo que aquellas prácticas de gestión curricular (gráfico 1) y su frecuencia de ejecución declarada por los JUTP, se evidencia que la práctica "Observación de la planificación curricular" presenta una frecuencia de realización en un 34,7\% en el rango muy a menudo y un $18,6 \%$ a menudo. Con un $46,7 \%$ declara realizar esta práctica sólo a veces durante el año académico.

La práctica "Monitoreo el trabajo en el aula", es realizada en un 87,7\% de los JUTP entre a veces y a menudo en el año escolar, de manera más frecuente o muy a menudo sólo un $6,6 \%$ y un $5,7 \%$ declara no haberlo realizado nunca.

La práctica de gestión que tienen mayor frecuencia de implementación, muy a menudo, por sobre el $30 \%$ es la promoción de la autonomía profesional en el trabajo de aula.

Las prácticas que se desarrollan de manera regular, a menudo y a veces, con mayor frecuencia son la planificación curricular en conjunto docentesJUTP con un 72\%; las acciones de cooperación conjunta entre docentes con un $87,7 \%$; la práctica de retroalimentación con un porcentaje de JUTP del $80 \%$; promover intencionadamente el trabajo colaborativo con un $81,6 \%$ de los JUTP con una frecuencia entre a veces y a menudo.

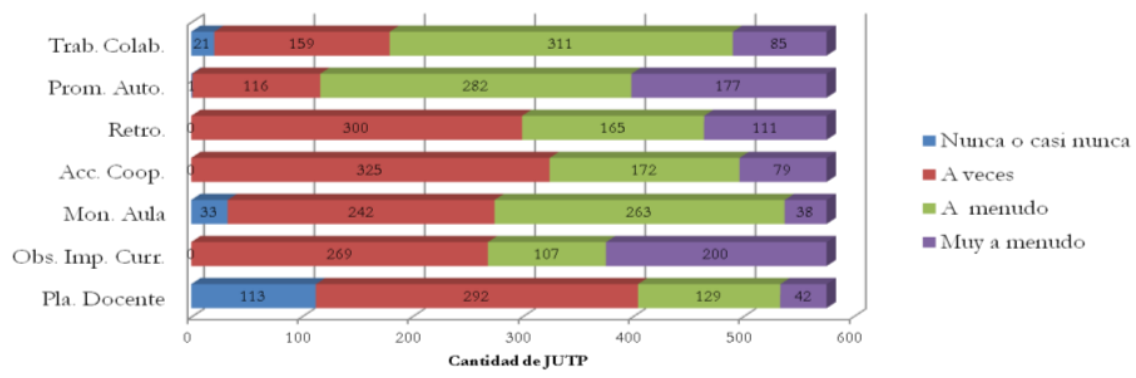

Gráfico 1: Implementación prácticas gestión curricular 
Ahora bien, cuando se preguntó sobre la profundización de las prácticas que implementan a través de las discusiones de las mismas y de diversos temas relacionados con las prácticas de gestión (gráfico 2), las cuales arrojan que con $47 \%$ de los JUTP realiza siempre una discusión con los profesores para mejorar las debilidades con respecto a la cobertura curricular, seguido por la discusión con respecto al aprendizaje de los estudiantes con un $42 \%$ y la generación de espacios para la reflexión pedagógica con un 36,6\%.

En el otro extremo están aquellas acciones que nunca las han realizado los JUTP en los establecimientos, como planes para el desarrollo profesional de los docentes con un 34\%, seguido de la modificación de tiempos pedagógicos de acuerdo a las necesidades del aprendizaje de los estudiantes con un $30,7 \%$ y otras acciones con similar porcentaje alrededor del $19 \%$ como lo son espacios de reflexión, cobertura curricular y clima de aula y aprendizaje.

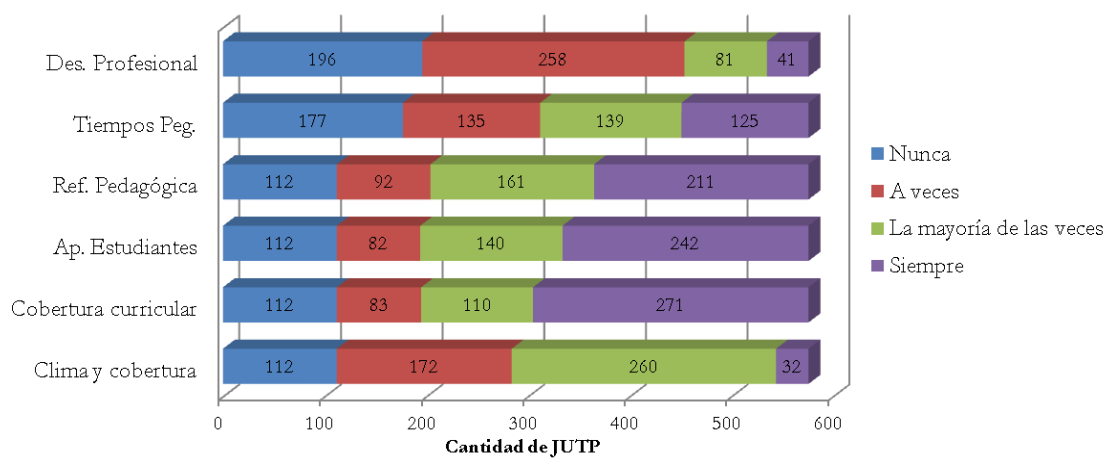

Gráfico 2: Temas de la implementación

\section{Sobre quienes implementan las prácticas de liderazgo}

En este ámbito, se realizó una reducción de aquellas prácticas que se implementan con mayor frecuencia en las organizaciones escolar, a saber: supervisión de la planificación curricular, observación en el aula, supervisión de la evaluación y entrega de retroalimentación. 
Los liderazgos que se involucran con estas prácticas pueden ser: director/a de la organización escolar, Jefe/a de la Unidad Técnica Pedagógica, Curriculista o Evaluador/a.

En relación a quién o quiénes son los que implementan las prácticas de gestión curricular en el establecimiento se tiene que (gráfico 3), en cuanto al proceso de "Observación en el aula" el 93,6\% de los JUTP señala que él o ella lleva a cabo dicha acción y sólo un $6,4 \%$ de los caos declara que los directores la realizan.

La implementación de la práctica "Retroalimentación docente" es realizada en un $98,1 \%$ por los JUTP y en un $1,6 \%$ por los directores/as. En el caso de la "Supervisión de la planificación curricular" está es realizada en un $81,8 \%$ por los JUTP y en un $18,2 \%$ por el curriculista de los establecimientos. Por su parte la "Supervisión de la evaluación" es implementada en un $73,8 \%$ por los JUTP y un $26,2 \%$ por el o la evaluadora de la unidad técnica pedagógica.

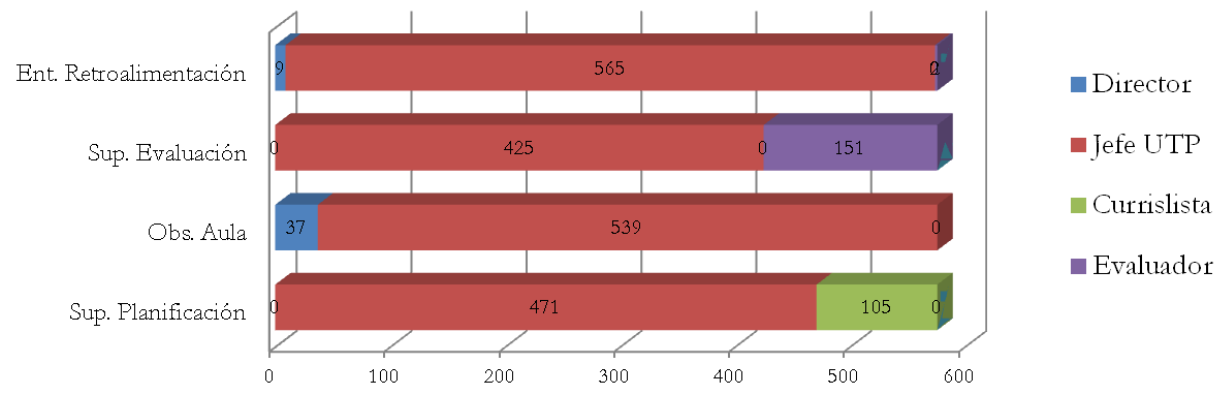

Gráfico 3: Frecuencia prácticas 


\section{Sobre la asociación de las prácticas de los líderes intermedios}

En este contexto, se observa en la Tabla 1, donde se realizó la correlación de las frecuencias de discusión que realizan los JUTP en distintos temas, encontramos cinco correlaciones entre variables, tres directas y dos inversas, el hecho que existan correlaciones entre las variables no implican que exista causalidad o dependencia entre ellas. En el caso de esta muestra nos encontramos que en ambos casos la intensidad de la relación es mínima, a pesar de ello nos entrega algunos indicios respecto de las posibles relaciones (directas o inversas) que pudiesen existir de las prácticas de los JUTP y las prácticas pedagógicas docentes.

Entre las variables que se evidencia una correlación directa esta la práctica de los JUTP "frecuencia de la discusión sobre la cobertura curricular" que presenta una relación mínima o muy baja con rho $=0,090$ y $\mathrm{p}=0,031$ con respecto a la calidad del análisis de clases que realizan los profesores de aula. Al analizar la significación, vemos que es de 0,031 nuevamente, por lo que, se cumple que sea menor a 0,05 lo que indica que la correlación que se ha establecido (mínima) es muy probablemente cierta.

Una segunda correlación directa, se establece con las variables de la "frecuencia de discusión sobre el clima y disciplina" realizada e intencionada por los JUTP y como es la evaluación de los profesores sobre la "atribución de los resultados de aprendizaje" de los estudiantes, esta relación, así como la anterior, es mínima con rho=0,082 y p=0,049. Y la última relación directa que encontramos es con el proceso de los JUTP, "frecuencia del monitoreo al aula" y el resultado de la dimensión "análisis de actividades de una clase" esta dimensión abarca otras prácticas docentes en las cuales incide el resultado general de la dimensión evaluada. Así, tenemos que la correlación entre ambas prácticas en baja o mínima con valor rho $=0,082$ y $\mathrm{p}=0,049$.

Si bien, los valores de intensidad de las correlaciones directas son mínimos en los dos últimos casos, la significancia nos da señales con respecto a que estas relaciones sean probablemente verdaderas. 
En el caso de las correlaciones inversas, estas las encontramos en la "frecuencia de discusión de la cobertura curricular" y "calidad de cierre de una clase", y por otra parte, la "frecuencia de discusión sobre el aprendizaje de los estudiantes" y "calidad de cierre de una clase". En el primer par de relaciones inversas los valores son $r h o=-0,096$ y $\mathrm{p}=0,022$. Mientras que en la segunda correlación los valores son $\mathrm{rho}=-0,086$ y $\mathrm{p}=0,038$ en ambos casos la relación inversa es mínima, sin embargo, el valor más de relación inversa es entre las variables "frecuencia de discusión de la cobertura curricular" y "calidad de cierre de una clase".

Tabla 1

Prácticas de gestión curricular y prácticas docentes

\begin{tabular}{|c|c|c|c|c|c|c|c|c|c|c|c|}
\hline & & 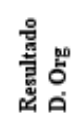 & 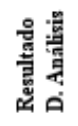 & 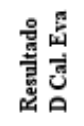 & 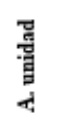 & 弯 & 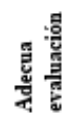 & 总 & 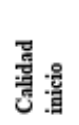 & 苞 & च 逆 \\
\hline \multirow{2}{*}{ F. planificación con docentes } & Rho &, 054 &,- 010 &, 002 &, 005 &,- 016 &, 010 &, 037 &, 074 &, 025 &, 067 \\
\hline & Sig. &, 197 &, 820 &, 954 &, 900 &, 702 &, 806 &, 371 &, 076 &, 557 &, 108 \\
\hline \multirow{2}{*}{$\begin{array}{l}\text { F. observación implantación } \\
\text { curricular }\end{array}$} & Rho &,- 009 &,- 022 &,- 013 &, 003 &,- 026 &,- 033 &,- 034 &, 023 &, 044 &,- 047 \\
\hline & Sig. & 824 &, 602 &, 761 & 941 &, 534 &, 433 & ,413 &, 587 &, 289 &, 255 \\
\hline \multirow[b]{2}{*}{ F. monitoreo aula } & Rho &, 030 &, $082^{*}$ &,- 028 &, 047 &, 074 &,- 026 &,- 007 &, 024 &, 010 &,- 015 \\
\hline & Sig. & ,469 & ,049 &, 507 &, 256 &, 076 &, 541 &, 869 &, 572 &, 818 &, 723 \\
\hline \multirow{2}{*}{$\begin{array}{l}\text { F. discusión } \\
\text { disciplina }\end{array}$} & Rho &,- 012 &, 006 &, 006 & 020 &, 066 &, 032 &, 032 &,- 070 &,- 080 &,- 070 \\
\hline & Sig. &, 772 &, 883 &, 880 &, 626 &, 114 &, 440 &, 437 &, 091 &, 054 &, 092 \\
\hline \multirow{2}{*}{$\begin{array}{l}\text { F. discusión } \\
\text { curricular }\end{array}$} & Rho &,- 042 & 080 &,- 015 &, 053 &, $090^{\circ}$ &, 022 &, 009 &,- 015 &,$- 096^{*}$ &, 003 \\
\hline & Sig. &, 316 &, 054 &, 715 & 202 & ,031 &, 599 &, 820 &, 712 &, 022 &, 949 \\
\hline \multirow{2}{*}{$\begin{array}{l}\text { F. discusión } \\
\text { estudiantes }\end{array}$} & Rho &,- 019 &, 028 &,- 006 &, 029 &, 008 &, 006 &, 049 &,- 043 &,$- 086^{*}$ &,- 024 \\
\hline & Sig. &, 642 &, 509 &, 890 &, 482 & 843 & ,891 &, 238 & 307 & ,038 &, 572 \\
\hline
\end{tabular}




\section{Discusiones}

Los hallazgos del estudio muestran que, los Jefes de Unidades Técnicas Pedagógicas, "líderes intermedios" de las organizaciones educativas implementan casi por completo las prácticas de los liderazgos pedagógicos, siendo por normativa las que deben implementar los directores de las instituciones.

A nivel transversal en los países de Latinoamérica, las funciones en los diversos casos se distribuyen entre las distintas áreas de la gestión y están completamente focalizadas en la figura del director de la organización escolar invisibilizando la figura de otros líderes o distribución del liderazgo, siendo la más débil aquella referida a la gestión pedagógica. En este sentido, aun cuando en la mayoría de los países el director tiene la función de dirigir, organizar y controlar el trabajo docente o la implementación del programa académico, las funciones de supervisión de aula, asesoría a los docentes y evaluación de su desempeño solo aparecen en un número menor de países, y principalmente en Argentina y Chile (UNESCO, 2014).

En este contexto, los líderes intermedios destinan el 70\% a tareas propias del currículo y la actividad docente, por ejemplo: observaciones de clases, retroalimentación docente, revisión de planificaciones y evaluaciones, y actividades de desarrollo profesional docente. Según la OCDE (2014), los directores de las escuelas en Chile dedican un $27 \%$ de tiempo a tareas de índole pedagógico. Evidenciando la carencia de esta figura directiva al interior de las instituciones y por otra se aprecia que las fortalezas del director son percibidas principalmente en su rol de rendir cuentas, administrar recursos o dirigir reuniones. Por otra parte, sus mayores debilidades radican en lo técnico-pedagógico: su manejo de las estrategias pedagógicas, de la evaluación de los aprendizajes, planificación de la enseñanza (Weinstein y Muñoz, 2012). Adicional a ello, también se ha reportado que la fiabilidad del juicio del director se ve seriamente mermada cuando en el ejercicio de su función predomina la dimensión administrativa por encima de la gestión pedagógica (Manzi et al., 2011). 
Por otra parte, cuando vemos las asociaciones entre prácticas de los líderes intermedios y aquellas que realizan los docentes nos encontramos que en el caso de la observación a la implementación curricular tiene una relación estadísticamente significativa con la práctica docente estructura de clases con $(\mathrm{X} 2=6,061$ y $\mathrm{p}=0,048)$, la calidad del cierre $(\mathrm{X} 2=9,070$ y $\mathrm{p}<0,011)$ y la calidad de la explicación entregada por los docentes en una clase $(\mathrm{X} 2=9,070$ y $\mathrm{p}<0,050)$, estas prácticas docentes están incluidas como indicadores en el sistema de evaluación docente. Si bien encontramos algunas prácticas relacionadas, esto no se da en todas ellas, por lo que llevaría necesariamente a generar una cultura y organización laboral en que el perfeccionamiento de las prácticas docentes a través del monitoreo que sirva para mejorar el trabajo docente $y$, a la postre, el aprendizaje (UNESCO, 2016) sumado a ello es que las prácticas docentes están relacionadas con factores tales como las convicciones docentes, las características de formación y el desarrollo profesional de los profesores (Vieluf, Kaplan, Klieme, y Bayer, 2012).

Así, cuando vemos los resultados de las prácticas en el contexto de la evaluación docente del año 2015 de ambas regiones, nos encontramos que en el caso de la práctica estructura de la clase un $63,2 \%$ de ellos obtiene el nivel mínimo esperado (competente), lo cual significa que cumple con lo requerido para ejercer profesionalmente el rol docente. Aun cuando no es excepcional, se trata de un buen desempeño (MINEDUC, 2016) y un 2.6 obtiene un nivel destacado. El resto de profesores se encuentra en niveles básico e insatisfactorio.

\section{Conclusiones}

El trabajo técnico-pedagógico, es el motor de la acción pedagógica de los docentes y directivos al interior de las organizaciones escolares. Para ello, al interior de los establecimientos deben existir liderazgos educativos en distintos niveles que promuevan dicha acción.

Podemos concluir que se la investigación arrojó, que en Chile se distinguen dos tipos de liderazgo al interior de los establecimientos 
educacionales: el director o directora de la organización "líder primario" y el o la Jefe de Unidad Técnico Pedagógica "líder intermedio".

Las prácticas de los JUTP como líderes intermedios son uno de los puntos centrales en la gestión pedagógica curricular (Beltrán, 2014), cuyo liderazgo lo asumen las denominadas Unidades Técnico Pedagógicas (Rodríguez, 2011; Mansilla y Miranda, 2011).

En este contexto, se puede concluir que los JUTP implementan todas y cada una de las prácticas de gestión curricular, a saber, con distintas frecuencias en cada una de ellas, entre las más implementadas son:

- Observación de la implementación curricular

- Retroalimentación docente

- Acciones de cooperación

- Promoción de la autonomía

- Discusiones con respecto a: aprendizaje de los estudiantes, cobertura curricular y clima en aula.

Así mismo, la implementación de las prácticas de gestión curricular (indistintamente la frecuencia) permite concluir que los líderes intermedios poseen una base de trabajo que podría sostener una articulación entre los planes y programas de estudio, la implementación de los mismos en la sala de clases y la adquisición de los aprendizajes por parte de los estudiantes, mediado a través de las prácticas de los docentes (Glattorn et al., 2013).

También se concluye que, la asociación de estos líderes en los establecimientos educativos no está documentada teórica y empíricamente. Sí nos encontramos con el impacto de los líderes escolares en el resultado de los estudiantes en diversos estudios de corte cualitativo (Portin et al., 2009; Penlington, Kington, y Day, 2008) y cuantitativos a gran escala (AligMielcarek y Hoy, 2005).

En base a lo anterior, se produciría una asociación de los líderes intermedios sobre las prácticas pedagógicas docentes en el contexto de la preparación de la enseñanza. Específicamente en los momentos del trabajo 
con la planificación y organización curricular, esto en base a que las pruebas estadísticas de asociación arrojaron un nivel de significancia de $\mathrm{p}=0,046$ cuando relacionamos la frecuencia sobre la discusión de la cobertura curricular y el nivel de desempeño del análisis de las actividades de clases que realizan los profesores. Esto nos permitiría concluir que, si los JUTP tuvieran una frecuencia promedio de 3 (la mayoría de las veces) en la implementación de esta práctica los docentes mejorarían sus niveles de desempeño en el análisis de sus clases.

En este mismo sentido, cuando correlacionamos ambas variables (frecuencia de discusión sobre la cobertura curricular y el análisis de clases) se tuvo que la intensidad es mínima rho=0,090 pero no descartable, con $\mathrm{p}=0,031$. Situación inversa, sucede cuando se correlacionó con la calidad del cierre que hacen los profesores en sus clases, ya que ahí rho=-0,096 y $\mathrm{p}=0,022$. Lo anterior, nos indica que los JUTP estarían proveyendo de recursos para el desarrollo profesional y trabajando directamente con los docentes de aula (Leithwood et al., 2009), especialmente en aspectos de asesoramiento técnico pedagógico.

\section{Referencias}

Alig-Mielcarek, J., y Hoy, W. (2005). Instructional leadership: Its nature, meaning, and influence. En C. G. Miskel y W. K. Hoy (Eds.), Educational leadership and reform (pp. 29-52). Greenwich, CT: Information Age.

Álvarez, M. (2003). La dirección escolar en el contexto europeo. Revista Organización Y Gestión Educativa, 2, 15-19.

Bellei, C., Vanni, X., y Valenzuela, J. (2015). Una mirada a los procesos de mejoramiento escolar en Chile. Santiago de Chile: CIAE.

Beltrán, J. (2014). Factores que dificultan la gestión pedagógica curricular de los jefes de unidades técnico pedagógica. Revista Mexicana de Investigación Educativa, 19(62), 939-961. 
106 Rodríguez \& Gairín - Prácticas de liderazgo intermedio

Bennet, J. (2009). An examination of the issues facing first time subject leaders during their first year of appointment in New Zealand secondary schools (Doctoral dissertation, Unitec Institute of Technology).

Bolívar, A. (2015). Un liderazgo pedagógico en una comunidad que aprende. Padres y Maestros, (361), 23-27.

Bolívar, A. (2010). El liderazgo educativo y su papel en la mejora. Psicoperspectivas, 9, 9-33.

Burch, P., y Heinrich, C. (2015). Mixed Methods for Policy Research and Program Evaluation. Thousand Oaks, California: SAGE.

Bush, T. (2011). Theories of educational leadership and management (4th ed.). London: SAGE.

Busher, H., y Harris, A. (2001). Subject leadership and school improvement. London: SAGE.

Comisión Europea/EACEA/Eurydice. (2013). Cifras clave del profesorado y la dirección de centros educativos en Europa. Edición 2013. Informe de Eurydice. Luxemburgo: Oficina de Publicaciones de la Unión Europea.

Corral, Y. (2009). Validez y confiabilidad de los instrumentos de investigación para la recolección de los datos. Revista Ciencias de la educación, Segunda etapa 1(33), 228-247.

Creswell, J. (2014). Research design: qualitative, quantitative, and mixed methods approaches (4th ed.). Thousand Oaks, California: SAGE.

Del Rincón, D., Arnal, J., Latorre, A. y Sans, A. (1995). Técnicas de investigación en ciencias sociales. Madrid: Dykinson.

Elmore, R. (2010). Mejorando las escuelas desde la sala de clases. Santiago de Chile: Fundación Chile.

Fitzgerald, T. (2009). The tyranny of bureaucracy continuing challenges of leading and managing from the middle. Educational Management Administration \& Leadership, 37(1), 51-65.

Flückiger, B., Lovett, S., Dempster, N., y Brown, S. (2015). Middle Leaders: Career pathways and professional learning needs. Leading \& Managing, 21(2), 60-74. 
Francis, O.B. y Oluwatoyin, F.C. (2019). Principals Personnel Characteristic Skills: A Predictor of Teachers' Classroom Management in Ekiti State Secondary School. International Journal of Educational Leadership and Management, 7(1), 72-103.

Gairín, J. (2003). Documento final de la Subcomisión de perfil y competencias del pedagogo en el siglo XXI. Comisión interdepartamental de Pedagogía. Bellaterra: Universidad Autónoma de Barcelona.

Galdames, S. y Rodríguez, S. (2010). Líderes educativos previos a cargos directivos: Una nueva etapa de formación. REICE. Revista Iberoamericana sobre Calidad, Eficacia y Cambio en Educación, $8(4), 50-64$.

García, E., y Cabero, J. (2011). Diseño y validación de un cuestionario dirigido a describir la evaluación en procesos de educación a distancia. Edutec. Revista Electrónica De Tecnología Educativa, (35), a156.

Glattorn, A., Boschee, F., Whitehead, B., y Boschee, B. (2013). Curriculum leadership. strategies for development and implementation (3th ed.). California: SAGE.

Grootenboer, P., Edwards-Groves, C., \&y Rönnerman, K. (2015). Leading practice development: Voices from the middle. Professional Development in Education, 41(3), 508-526.

Hargreaves, A. y Fullan, M. (2014). Capital profesional. Transformar la enseñanza en cada escuela. Madrid: Morata.

Hernández, R., Fernández, C. y Baptista, P. (2014). Definición del alcance de la investigación que se realizará: exploratorio, descriptivo, correlacional o explicativo. En Hernández, R., Fernández, C. y Baptista, P. Metodología de la Investigación (6 ed., págs. 88-101). México: McGraw-Hill.

Leithwood, K. (2016). Department-Head Leadership for School Improvement. Leadership and Policy in Schools, 15(2), 117 - 140.

Leithwood, K., Mascall, B., y Strauss, T. (2009). Distributed leadership according to the evidence. London: Routledge. 
Leithwood, K., Day, C., Sammons, P., Harris, A., y Hopkins, D. (2006). Successful school leadership: What it is and how it influences pupil learning. Nottingham: N. C. for S. Leadership y D. for E. and Skills. Leithwood, K., y Levin, B. (2005). Assessing School Leader and Leadership Programme Effects on Pupil Learning. Nottingham: Department for Education and Skills.

Male, T., y Palaiologou, I. (2012). Learning-centred leadership or pedagogical leadership? An alternative approach to leadership in education contexts, International. International. Journal of Leadership in Education, 15 (1), 107-118.

Mansilla, S., y Miranda, J. (2011). Las unidades técnicas pedagógicas en la enseñanza media: razón administrativa y razón pedagógica en contextos secundarios vulnerables. In Congreso Iberoamericano de Educación. Metas 2021 (pp. 1-11). Buenos Aires. Recuperado de www.chubut.edu.ar/descargas/secundaria/congreso/DOCENTES/R1 1 45_Mansilla.pdf

Manzi, J., González, R., y Sun, Y. (2011). La evaluación docente en chile. Santiago de Chile: MIDE UC.

MINEDUC. (2016). Resultados de la evaluación docente 2015. Santiago de Chile: CPEIP.

MINEDUC. (2015). Marco para la buena dirección y liderazgo escolar. Santiago de Chile: CPEIP.

Murphy, J., Elliott, S., Goldring, E., y Porter, A. (2007). Leadership for Learning. School Leadership and Management, 27 (1), 170-201.

Nuñez, I., Weinstein, J., y Muñoz, G. (2010). ¿Posición Olvidada? una Mirada Desde la Normativa a la Historia de la Dirección Escolar en Chile. Psicoperspectivas. Individuo Y Sociedad, 9 (2), 53-81.

OCDE. (2014). Estudio Internacional de la enseñanza y el aprendizaje. Informe Chileno. París: OCDE.

OCDE/CEPPE. (2013). Learning Standards, Teaching Standards and Standards for School Principals: A Comparative Study (Education Working Papers No. 99). París: OCDE. 
Penlington, C., Kington, A., y Day, C. (2008) Leadership in Improving Schools: A Qualitative Perspective. School Leadership and Management, Special Issue: The impact of school leadership on student outcomes, 28 (1), 65-82.

Pinto, V., Galdames, S. y Rodríguez, S. (2010). Aprendizajes y desafíos para la formación de líderes intermedios. Psicoperspectivas. Individuo y Sociedad, 9(2), 136-157.

Pont, B., Nushe, D., y Moorman, H. (2008). Mejorar el liderazgo escolar. Volumen 1: política y práctica. París: OCDE.

Porkka, S. (2014). Development discussions as a part of pedagogical management. Keveko: Facultad de Educación, Universidad de Turku.

Portin, B., Knapp, M., Dareff, S., Feldman, S., Russell, F., Samuelson, C. y Yeh, T. (2009). Leadership for learning improvement in urban school. Seattle: University of Washinton.

Rodríguez, G. (2011). Funciones y rasgos del liderazgo pedagógico en los centros de enseñanza. Educación y Educadores, 14 (2), 253-267.

Rodríguez, G. y Gairín, J. (2017). Influence of the practices of pedagogical leadership in the educational pedagogical practices: case in Chile of the Pedagogical Technical Units. International Journal of Educational Leadership and Management, 5(1), 6-29. doi: 10.17583/ijelm.2017.2469

Sans-Martín, A., Guàrdia, J., y Triadó-Ivern, M. (2016). El liderazgo educativo en Europa: una aproximación transcultural. Revista de Educación, 371 (Enero-Marzo), 83-106.

Supovitz, J., Sirinides, P. y May H. (2009). How principal and peers influence teaching and learning. Educational Administration Quarterly, 46(1), 31-56.

Tejada, J. y Giménez, V. (2006). Formación de formadores. Escenario instituciona. Madrid: Thomson.

Törnsén, M. (2014). Classroom observations and supervision - essential dimensions of pedagogical leadership. International Journal of Educational Management, 28 (7), 856-868. 
110 Rodríguez \& Gairín - Prácticas de liderazgo intermedio

Triviños, A. (2013). Prácticas de liderazgo distribuido en el contexto escolar. Estado del arte. Santiago de Chile: Pontificia Universidad Católica de Chile.

UNESCO. (2016). Tercer Estudio Regional Comparativo y Explicativo TERCE. Recomendaciones de políticas educativas en América Latina. Santiago de Chile: UNESCO/OREALC.

UNESCO. (2014). El liderazgo escolar en América latina y el Caribe: un estado del arte con base en ocho sistemas escolares de la región. Santiago de Chile: UNESCO/OREALC.

Vicente de Vera, M.I. y Gabari, M.I. (2019). Liderazgo Pedagógico en Educación Secundaria: Aportaciones desde la Evaluación de Burnout-Resiliencia en Docentes. International Journal of Educational Leadership and Management, 7(1), 104-134.

Vieluf, S., Kaplan, D., Klieme, E., y Bayer, S. (2012). Teaching Practices and Pedagogical Innovation: Evidence from TALIS. París: OCDE.

Wahlstrom, K. y Seashore, K. (2012). How Teachers Experience Principal Leadership: The Roles of Professional Community, Trust, Efficacy, and Shared Responsibility. Educational Administration Quarterly 44(4), 458-495.

Weinstein, J., y Hernández, M. (2015). ¿Un centro nacional de liderazgo escolar en Chile? Antecedentes para su diseño. Santiago de Chile: Espacio Público.

Weinstein, J., y Muñoz, G. (2012). ¿Qué sabemos sobre los directores de escuela en Chile? Santiago de Chile: Centro de Innovación en Educación de Fundación Chile y Centro de Estudios y Políticas y Prácticas en Educación.

Weller, D. (2001). Department Heads: The Most Underutilized Leadership Position. NASSP Bulletin, 85(625), 73-81.

York-Barr, J. y Duke, K (2004). What do we know about teacher leadership? Findings from two decades of scholarship. Review of Educational Research, 74(3), 225-316. 
Guillermo Andrés Rodríguez Molina Profesor de EGB, Máster en Psicología y Doctor en Educación, actualmente es jefe de la Unidad de Calidad Pedagógica de la Direccion de Educacion Municipal de la Comuna de Coronel-Chile. Ha sido docente de la cátedra de curriculum y práctica de la Universidad de Concepcion. Docente capacitador de profesorado de distintas comunas en el área de gestión de curricular de aula.

Contact Address: Janequeo 1510 Depto. 601 T1, Concepción Chile, CP: 4081854

E-mail: guiandres.rm@gmail.com

Joaquín Gairín Sallán Profesor de EGB, Graduado social, Psicólogo y Pedagogo. Catedrático de Didáctica y Didáctica y Organización escolar. Su experiencia docente e institucional es amplia, habiendo sido director de centro educativo, decano de facultad, comisionado del Clúster en Educación y Formación, director de departamento universitario y del ICE de la UAB. Consultor internacional de programas de reforma educativa en España y Latinoamérica. Actualmente, dirige proyectos sobre desarrollo social y educativo, desarrollo organizacional, procesos de cambio educativo, liderazgo, evaluación de programas e instituciones, TIC en formación y evaluación de impacto.

Contact Address: Departament de Pedagogia Aplicada, Edifici G6-247 Campus de Bellaterra, 08193, Bellaterra (Cerdanyola del Vallès)

E-mail: joaquin.gairin@uab.cat 